\title{
Short-bunch extraction in a rapid cycling synchrotron
}

\author{
Ye Zou, ${ }^{1,2}$ Jingyu Tang, ${ }^{2,1,}$ Jinfang Chen, ${ }^{2}$ Xiao Li, ${ }^{2}$ and Hong Sun ${ }^{2}$ \\ ${ }^{1}$ University of Science and Technology of China, Hefei, Anhui 230029, People's Republic of China \\ ${ }^{2}$ Institute of High Energy Physics, CAS, Yuquan Road 19B, Beijing 100049, People's Republic of China
}

(Received 6 March 2014; published 17 June 2014)

\begin{abstract}
Short bunch beams of high intensity are required by many applications, and they are usually obtained by using bunch compression before the extraction in relatively slow cycling synchrotrons or accumulator rings. In this article, short bunch extraction by the bunch compression method is proposed to apply to a rapid cycling synchrotron (RCS) where the time duration is very limited to exploit the bunch compression process. The method is practically applied in the RCS of the China Spallation Neutron Source (CSNS) to obtain short-bunch beams for white neutron applications. Different short-bunch extraction scenarios have been studied, from keeping full beam power to reduced beam powers. A special desynchronization method is designed to solve the problem of slow acceleration during the bunch rotation process when the magnetic field is still ramping up. The space charge effect and beam loading effect during the bunch compression have also been taken into account in this high-intensity RCS. Multiparticle simulations have been carried out to show the effectiveness of the method with the input of the CSNS RCS. With a sacrifice of about twothirds of the beam power, the rms bunch length of the extracted beam can be reduced to about one-ninth of the one in the nominal operation mode.
\end{abstract}

DOI: 10.1103/PhysRevSTAB.17.060101

PACS numbers: 29.20.dk, 29.27.Ac, 29.27.Bd

\section{INTRODUCTION}

Short-bunch high-intensity hadron beams are required by many applications such as the production of neutrino beams and radioactive ion beams and white neutron sources. The bunch compression method just before fast beam extraction has been proposed to obtain very short bunch beams from synchrotrons. This is to use the combination of adiabatic bunch lengthening and the nonadiabatic bunch rotation processes. This method is relatively easy to realize in slow-cycling synchrotrons or accumulator rings where the whole extraction process is executed without beam acceleration [1-4]. In a rapid cycling synchrotron (RCS), the magnetic field ramping is usually driven by a resonant power supply and no evident flat-top field can be provided for applying the nonadiabatic bunch compression method. For example, the RCS type proton driver studied by the Neutrino Factory considered using adiabatic bunch compression [5]. In this article, the method to apply the nonadiabatic bunch compression method in such RCSs is presented. The method is also applied to the RCS of the China Spallation Neutron Source (CSNS) for the application of white neutrons.

CSNS is a multidisciplinary research facility under construction [6], which is to be built in two or three

\footnotetext{
*Corresponding author. tangjy@ihep.ac.cn
}

Published by the American Physical Society under the terms of the Creative Commons Attribution 3.0 License. Further distribution of this work must maintain attribution to the author(s) and the published article's title, journal citation, and DOI. phases. In the first phase, the RCS is to accelerate beams from $80 \mathrm{MeV}$ to $1.6 \mathrm{GeV}$ in a repetition rate of $25 \mathrm{~Hz}$, and the extracted beam power is $100 \mathrm{~kW}$. Figure 1 and Table I show the layout and some main parameters of the RCS.

The backstreaming neutrons from the spallation target of CSNS are very strong and harmful to the devices in the proton beam transport line if they are not well treated [7]. However, the studies found that the neutron beam has

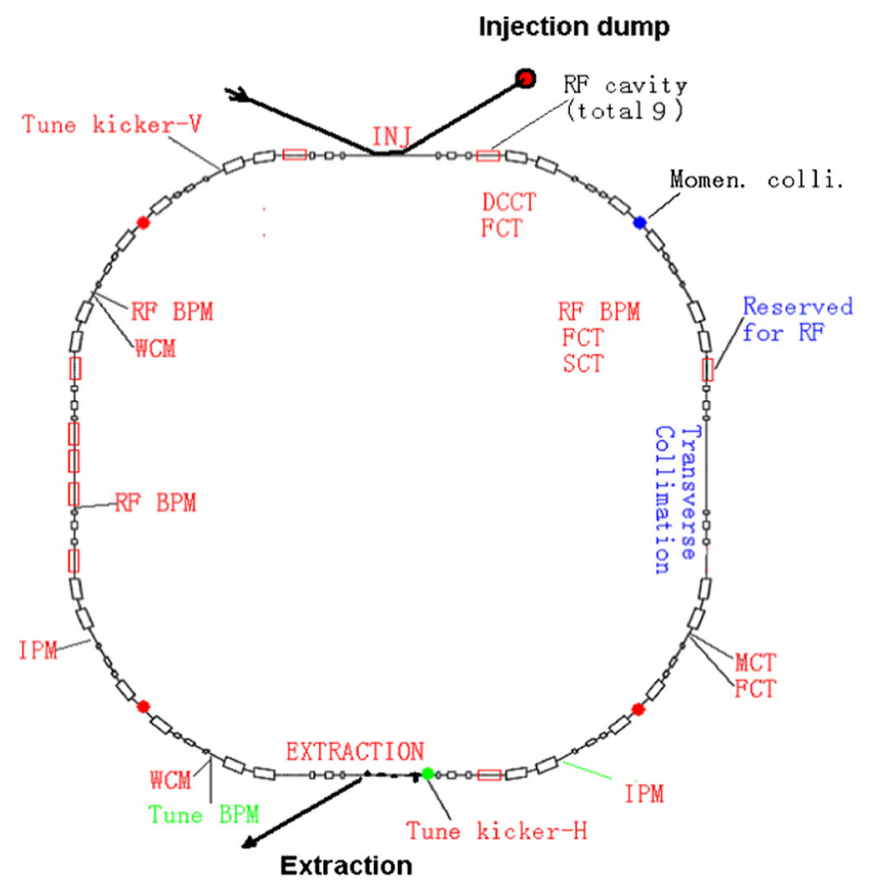

FIG. 1. Layout of the CSNS RCS.

$1098-4402 / 14 / 17(6) / 060101(9)$

060101-1

Published by the American Physical Society 
TABLE I. Some parameters of the CSNS accelerator complex at phase I.

RCS circumference (m)

RCS injection energy $(\mathrm{GeV})$

RCS extraction energy $(\mathrm{GeV})$

RCS extraction beam power $(\mathrm{kW})$

RCS maximum rf voltage $(\mathrm{kV})$

RCS betatron tunes $(\mathrm{h} / \mathrm{v})$

RCS rf harmonics

RCS phase slippage at extraction

RCS transverse acceptance $(\pi \mathrm{mm} \mathrm{mrad})$

RCS collimation acceptance $(\pi \mathrm{mm} \mathrm{mrad})$

Linac beam peak current (mA)

Linac rf duty factor

Linac beam momentum spread after debunching

Linac beam transverse emittance after collimation $(\pi \mathrm{mm}$ mrad $)$

excellent properties in energy spectrum and time structure, and it has been proposed to build a white neutron beam line or white neutron source (WNS) for nuclear data measurements [8], which is now under construction. The neutron pulse length is critical in defining the neutrons' energy by the time-of-flight method, and should be reduced to be as short as possible for some high-precision measurements. The time structure of the backstreaming neutrons is jointly determined by the pulse length of the impinging proton beam and the thickness of the spallation target. Simulations show that the target thickness dominates the time resolution for the lower energy part of the wide-range energy neutrons, while the proton pulse length dominates for the higher energy part. As the target length cannot be changed for this application, it would be interesting to shorten the proton pulse length by designing special dedicated working modes for the CSNS accelerators. This objective drives the studies presented in the article.

\section{SHORT BUNCH EXTRACTION METHODS}

\section{A. Principle of the bunch compression method}

In small-amplitude or linear synchrotron motion approximation, the rms bunch length and height $\sigma_{\tau}$ and $\sigma_{\delta}$ in a matched rf bucket are expressed by [9]

$$
\begin{gathered}
\sigma_{\tau}=\sqrt{\frac{A_{\mathrm{rms}}}{\omega_{0}}}\left(\frac{2|\eta|}{\pi h e V \beta^{2} E\left|\cos \varphi_{s}\right|}\right)^{\frac{1}{4}}, \\
\alpha_{\delta}=\sqrt{\frac{\omega_{0} A_{\mathrm{rms}}}{\pi \beta^{2} E}}\left(\frac{h e V\left|\cos \varphi_{s}\right|}{2 \pi \beta^{2} E|\eta|}\right)^{\frac{1}{4}},
\end{gathered}
$$

where $A_{\mathrm{rms}}, \omega_{0}, \eta, h$, and $\varphi_{s}$ denote the invariant rms phase space area in eV-s, revolution frequency of the reference particle, phase-slip factor, harmonic number, and synchronous phase, respectively. From Eq. (1), one can see that the bunch length is inversely proportional to $V^{1 / 4}$, so it is not effective by directly increasing the voltage to shorten the bunch length. A more effective method called the bunch compression method makes more complicated bunch gymnastics [10], which uses a combination of adiabatic bunch lengthening and nonadiabatic bunch rotation processes. Suppose the rf voltage changes from the nominal setting $V_{i}$ down to a minimum value $V_{1}$ adiabatically, and then up to the maximum available value $V_{2}$ nonadiabatically. According to Eq. (1), the extension ratio of the rms bunch length during the adiabatic process is equal to

$$
r_{c \max , V_{i} \rightarrow V_{1}}=\frac{\sigma_{\tau, i}}{\sigma_{\tau, m}}=\left(\frac{V_{1}}{V_{i}}\right)^{\frac{1}{4}},
$$

where $\sigma_{\tau, i}$ and $\sigma_{\tau, m}$ denote the initial bunch length and the medium-stage bunch length, respectively.

After the nonadiabatic rf voltage change from $V_{1}$ to $V_{2}$, the bunch performs rigid quadrupole mode oscillations or bunch rotation. After $1 / 4$ of the synchrotron period, the rms bunch length reaches the minimum and can be expressed by

$$
\sigma_{\tau, f}=\sqrt{\frac{A_{\mathrm{rms}}}{\omega_{0}}}\left(\frac{2|\eta|}{\pi h \beta^{2} E\left|\cos \varphi_{s}\right|}\right)^{\frac{1}{4}} \frac{\left(e V_{1}\right)^{1 / 4}}{\left(e V_{2}\right)^{1 / 2}},
$$

where $\sigma_{\tau, f}$ denotes the final bunch length. The compression ratio of the rms bunch length from $V_{1}$ to $V_{2}$ can be expressed by

$$
r_{c \max , V_{1} \rightarrow V_{2}}=\frac{\sigma_{\tau, m}}{\sigma_{\tau, f}}=\left(\frac{V_{2}}{V_{1}}\right)^{\frac{1}{2}} .
$$

The total compression ratio of the rms bunch length from the initial voltage $\left(V_{i}\right)$ to the low voltage $\left(V_{1}\right)$ and then to the final voltage $\left(V_{2}\right)$ can be expressed as

$$
R_{c \max , V_{i} \rightarrow V_{1} \rightarrow V_{2}}=\frac{\sigma_{\tau, i}}{\sigma_{\tau, f}}=\left(\frac{V_{1}}{V_{i}}\right)^{\frac{1}{4}}\left(\frac{V_{2}}{V_{1}}\right)^{\frac{1}{2}} .
$$

The bunch compression ratio depends only on the rf voltage ratios according to Eq. (6). From Eq. (6) one can see that the lower the initial voltage $\left(V_{i}\right)$ and the minimum voltage $\left(V_{1}\right)$ and the higher the final voltage $\left(V_{2}\right)$, the larger the bunch compression ratio and the shorter the length of the bunch.

However, in practice, there are limitations to apply the bunch compression method. In a high-intensity RCS, one has to consider the available time duration for executing the bunch compression process, the space charge effects during the bunch rotation process, and the beam loading effect. The space charge effect may become strong when the bunch shortens sharply during the bunch rotation. The initial beam emittance before the bunch compression is also determined by the beam dynamics at the lower energy of 
the acceleration cycle, due to the space charge effects and the longitudinal microwave instability. The beam loading effect means that the circulating beam current detunes the rf cavities and becomes very important when the RF voltage is very low. To avoid the instability in the low-level rf control system, the ratio of the circulating beam current and the driving current from the RF amplifier should be controlled below a certain value, e.g. 5. This limits the injection $\mathrm{rf}$ voltage and the lowest $\mathrm{rf}$ voltage for $V_{1}$. The accumulated particles should be decreased to ease the above problems, and this leads to the reduction of the extracted beam power. Different bunch chopping factors and beam powers are considered for the dedicated short-bunch operation modes.

\section{B. Desynchronization method}

As mentioned in Sec. I, different from its usual applications in compressor rings $[11,12]$ or slow cycling synchrotrons [13], where the bunch compression can be executed with a stationary beam, the situation in an RCS has to deal with beam acceleration during the bunch compression process. Because the magnetic field is usually driven by a resonant power supply and has a sinusoidal curve, the beam is still in slow acceleration when approaching the extraction. If the bunch rotation is performed in this condition, the asymmetric rf bucket will deform the bunch shape and increase the final bunch length. One can place the whole bunch rotation process around the maximum magnetic field to minimize the acceleration effect, though it will delay the extraction moment slightly. Here, a special desynchronization method is proposed to make sure that the rf bucket is always symmetric and the bunch is also centered at a zero-degree if phase during the bunch rotation process. With this desynchronization mode, when the adiabatic bunch lengthening starts, the rf frequency and phase are debounded from the magnetic field, namely, the rf bucket becomes stationary while the magnetic field is still ramping. To avoid the dipole oscillation, the rf phase is made smoothly from $7.6^{\circ}$ to zero. For comparison, the rf phase increases with the decaying rf voltage in the synchronization mode. As the momentum difference from the nominal one is very small when the beam is close to the extraction, the closed-orbit distortion due to the nonsynchronization is acceptable. One can make a simple evaluation of the nonsynchronism as follows.

The relative field difference between the moments of the desynchronization beginning and the peak field arrival is given by

$$
\frac{\Delta B}{B}=\frac{B_{A C}}{B_{\max }}[1-\cos (2 \pi \Delta t / T)],
$$

where $B_{A C}$ and $B_{\max }$ denote the amplitude of the ac magnetic field and the peak magnetic field, and $T$ and
$\Delta t$ denote the cycling period and the time difference between the two moments.

The relative difference between the beam momentum and the reference momentum corresponding to the magnetic field is given by $\Delta p / p=\Delta B / B$. Since $\Delta t / T$ is very small, one can make a simplification to Eq. (7), and then the closed-orbit distortion is defined by the dispersion function:

$$
\Delta x=D_{x} \frac{\Delta p}{p} \approx 2 \pi^{2} D_{x} \frac{B_{A C}}{B_{\max }}\left(\frac{\Delta t}{T}\right)^{2},
$$

Taking the example of the CSNS RCS, $B_{A C}=0.4081 \mathrm{~T}$, $B_{\max }=0.9808 \mathrm{~T}, D_{\mathrm{x}, \max }=3.38 \mathrm{~m}, \quad T=40 \mathrm{~ms}, \Delta t=$ $0.58 \mathrm{~ms}$, one can obtain $\Delta x_{\max }=5.8 \mathrm{~mm}$. This is considered acceptable, as the RCS has redundant room in the aperture at the extraction energy compared with that at the injection energy due to the shrinking of the transverse emittance and momentum spread during acceleration, which is usually more than $20 \mathrm{~mm}$.

\section{Short-bunch extraction at the CSNS RCS}

There are two bunches per pulse in the extracted proton beam in the normal operation mode of the CSNS RCS. The bunch length is determined by the rf voltage pattern and the longitudinal emittance which is defined by the injection scheme. As mentioned above, compared with the simple method by designing a high rf voltage at the extraction, the bunch compression method is more effective in shortening the bunch length for the extracted beam. The painting or phase space filling in the longitudinal plane is usually optimized to alleviate the space charge effects in such a high-intensity synchrotron. Here it should also be optimized to have a small emittance as a balance. A lower rf voltage at injection can make the emittance of the initial bunch smaller. However, if the voltage is too low, there will be problems for the rf bucket to contain the beam with some margin in the presence of a strong space charge. Another limitation factor for lowering the rf voltage is the beam loading effect, which is very important for the stable operation of the rf system [14-16]. In addition, in order to obtain small longitudinal emittance and avoid severe emittance dilution, one should use a smaller chopping factor for the injection which means a smaller duty factor within a linac pulse. This can be realized by changing the setting of the chopper in the low-energy beam transport (LEBT) section of the linac [17]. In the normal operation mode, the chopping factor is $50 \%$; in the dedicated mode for a short-bunch beam for white neutron applications, a $30 \%$ chopping factor can be used. At the same time, the injection with an on-momentum beam instead of an offmomentum beam [18] is used. However, with the reduced longitudinal emittance, the transverse space charge effects become more important. One can solve this problem by injecting less particles in a pulse, but this means the reduction of beam power. This is considered acceptable 


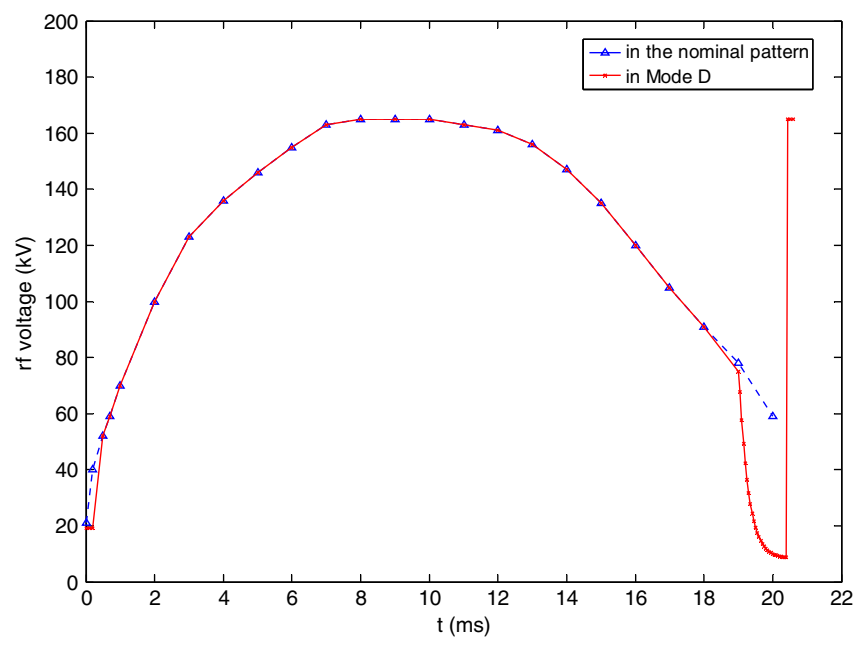

FIG. 2. Radio frequency voltage patterns of the nominal operation mode and the operation mode D with bunch compression (defined in Sec. III). Blue dotted line with triangles: the nominal rf voltage pattern; red solid line with crosses: rf voltage pattern in mode D.

for the dedicated mode of the white neutron source which will be operated in very limited periods per year. The details are given in the next section.

Once a small longitudinal emittance is formed during the injection, the following acceleation process is almost the same as in the nominal operation mode. Just before the extraction, e.g. $2 \mathrm{~ms}$ ahead of the peak magnetic field, the rf pattern is modified to apply the bunch compression method which is as follows (as shown in Fig. 2): (1) Decreasing the rf voltage adiabatically from the nominal high level to a very low one which is mainly limited by the bunch emittance and the beam loading effect, during which the bunch becomes gradually flat to follow the change of the rf bucket. The miminum period for the quasiadiabatic bucket shrinking is about $0.5 \mathrm{~ms}$. (2) Either from the beginning or in the middle of the bunch lengthening process, the desynchronization is applied to the $\mathrm{rf}$ system and the rf phase is smoothly moved to the bunch center. (3) Then the rf voltage is quickly or nonadiabatically increased to the maximum avaiable level of about $165 \mathrm{kV}$ in a period of about dozens of $\mu \mathrm{s}$ and the mismatched bunch will rotate in the enlarged rf bucket. (4) After a quarter of a synchrotron period, the bunch length will reach the minimum and then the single-turn extraction can be applied.

\section{OPERATION MODES AND SIMULATION RESULTS}

Taking the CSNS RCS parameters and the scheme for short-bunch extraction, multiparticle simulations with space charge with the ORBIT code [19] have been carried out to show how effective the method in realistic cases is. As the transverse motions have only averaged effect to the longitudinal motion, one needs only to perform simulations in 1D longitudinal direction. The transverse motions are kept in mind only by maintaining reasonable tune shifts that limit the choosing of the bunching factor. Different injection scenarios and beam powers are studied in the simulations. They are (1) mode-A: the nominal injection mode which delivers $100 \mathrm{~kW}$ in beam power and produces relatively long bunches; (2) mode-B: the modified injection mode which delivers a $50-\mathrm{kW}$ beam with shorter bunches; (3) mode-C: the dedicated short-bunch mode which delivers a $30-\mathrm{kW}$ beam with quite short bunches; (4) mode-D: the alternative dedicated short-bunch mode which delivers a 30-kW beam with very short bunches. For each injection mode, the extractions with bunch compression and without bunch compression are compared. Similar to Ref. [20], chopped beams are used for the injection and the rf bucket is made stationary during the injection. Except for mode-D where the chopping factor of $30 \%$ is used, the nominal chopping factor of $50 \%$ is used. For modes B, C, and D, the injection is modified from the nominal one in order to reduce the longitudinal emittance. A slight momentum deviation of $0.1 \%$ is used for the nominal injection mode and the on-momentum injection is used for the other modes. The number of macroparticles used in the simulations is 20000 . The longitudinal impedance has not been included in the simulations and will be studied later. The first estimate gives that it has much less influence than the longitudinal space charge effect in the CSNS RCS, especially when the beam power is reduced in the dedicated WNS operation modes.

Although lower rf voltage is preferred to obtain smaller longitudinal emittance at the injection and better bunch compression at the extraction, as mentioned before, the minimum rf voltage to apply is limited by the beam loading effect and other effects. We can make estimates about the lower limits of rf voltage by only considering the beam loading effect. This means that the lowest rf voltages are dependent on the beam power and the cavity impedances at the injection and extraction. Assuming a maximum beam loading factor of 5 to ensure the rf system stability, Table II shows the lowest rf voltages with different injection modes. However, we found that a very low injection voltage is not helpful in reducing further the longitudinal emittance due to the fast rf voltage ramping up after the injection which is nonperfect adiabatic and may produce emittance dilution. For the extraction, there is a similar situation. One needs

TABLE II. Radio frequency voltage lower limits by the beam loading effect and the applied values for different beam powers.

\begin{tabular}{lccc}
\hline \hline $\begin{array}{l}\text { Injection } \\
\text { mode }\end{array}$ & $\begin{array}{c}\text { Beam } \\
\text { power } \\
(\mathrm{kW})\end{array}$ & $\begin{array}{c}\text { Lowest rf voltage } \\
\text { at the injection/ } \\
\text { applied }(\mathrm{kV})\end{array}$ & $\begin{array}{c}\text { Lowest rf voltage } \\
\text { at the extraction/ } \\
\text { applied }(\mathrm{kV})\end{array}$ \\
\hline $\mathrm{A}$ & 100 & $10.7 / 21$ & $23 / 29$ \\
$\mathrm{~B}$ & 50 & $5.3 / 19$ & $11.5 / 14.5$ \\
$\mathrm{C} / \mathrm{D}$ & 30 & $3.2 / 19$ & $6.9 / 8.7$ \\
\hline \hline
\end{tabular}



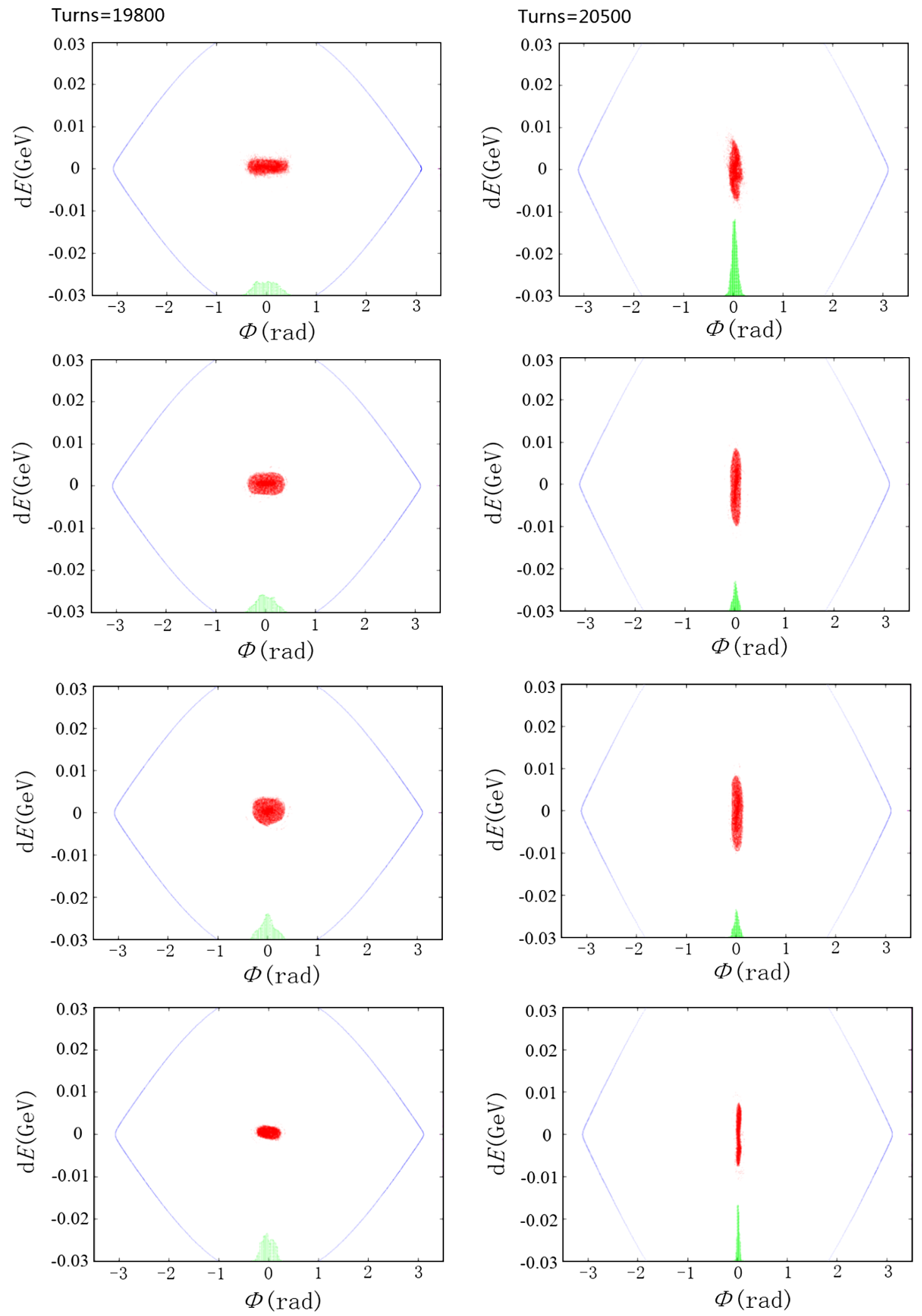

FIG. 3. Longitudinal beam distributions just before the extraction with different beam powers and chopping factors (Left: without bunch compression. Right: with bunch compression. Top to bottom represent modes A, B, C, and D, respectively.) 
more time to adiabatically decrease the rf voltage to a very low level as required by the bunch lengthening process, which is not obvious in a RCS. Therefore, some compromise has been made in choosing the injection rf voltages and $V_{1}$ at the extraction. The applied rf voltages in the simulations are also shown in Table II. The maximum rf voltage $\left(V_{2}\right)$ at the end of the nonadiabatic process or just before the bunch rotation starting is taken as $165 \mathrm{kV}$ which is the maximum available rf voltage for stable operation.

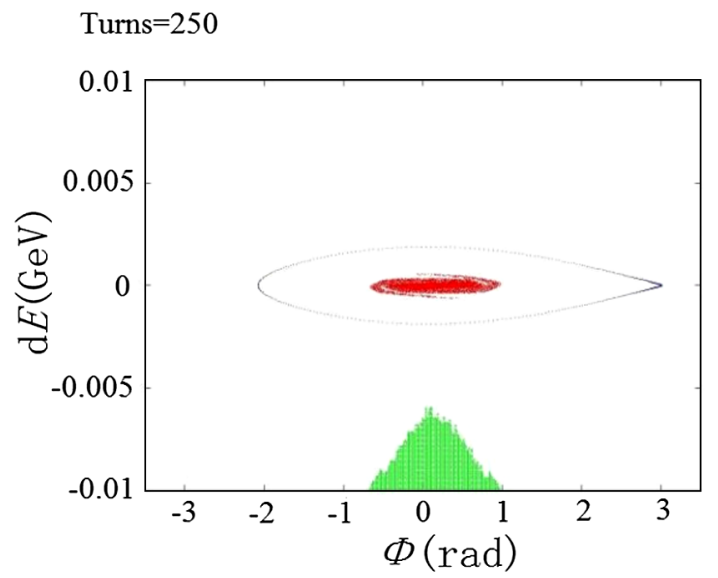

Turns $=20250$

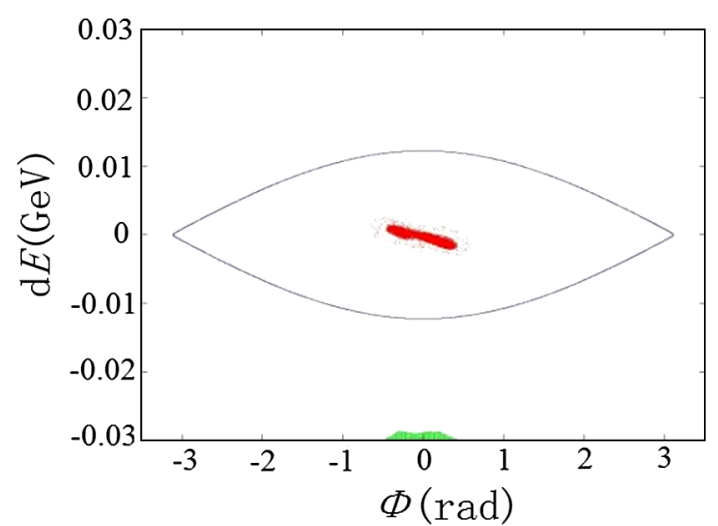

Turns $=20400$

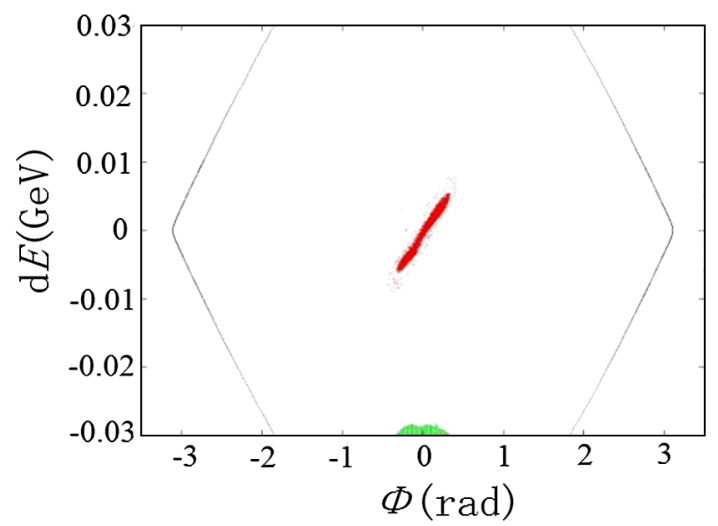

The rf voltage pattern with the injection mode $\mathrm{D}$ and the bunch rotation method is shown in Fig. 2. To ramp up the rf voltage in dozens of microseconds produces a nonlinear effect in the ferrite toroids of the rf cavities as shown in the rf tests of the prototype, and the tracking error in the voltage becomes significantly larger than the nominal $1 \%$. However, a tracking error of a few percent in rf voltage has almost no effect to the bunch rotation, and thus is tolerated.

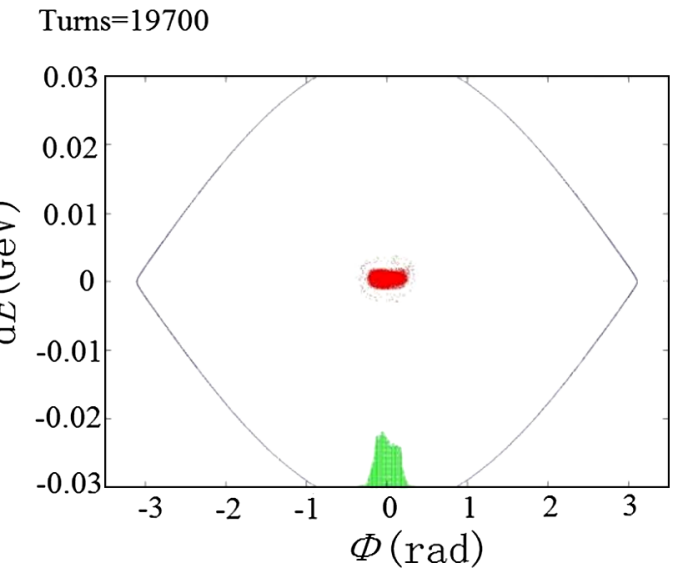

Turns $=20300$

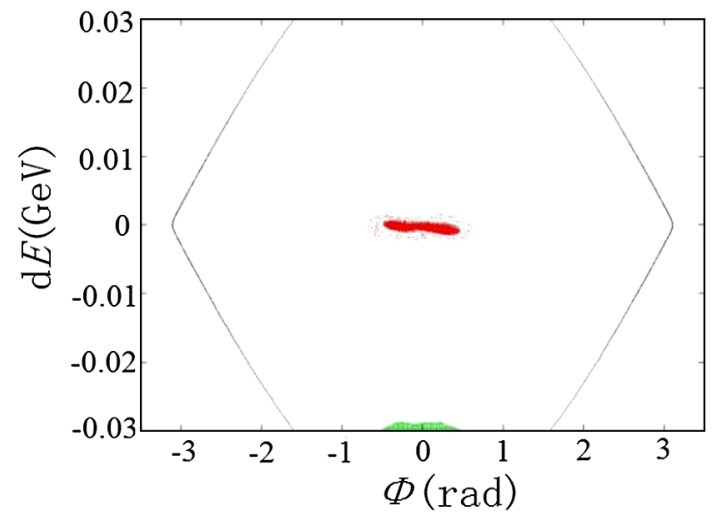

Turns $=20500$

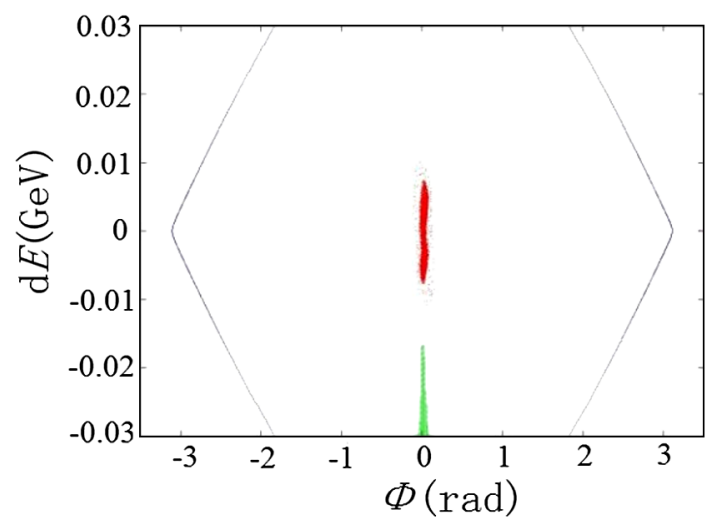

FIG. 4. Longitudinal beam distributions during the bunch compression process in the case of mode D. (Upper left to lower right, lines correspond to the moments of the end of the injection, beginning and end of the adiabatic process, beginning, middle, and end of the bunch rotation, respectively.) 

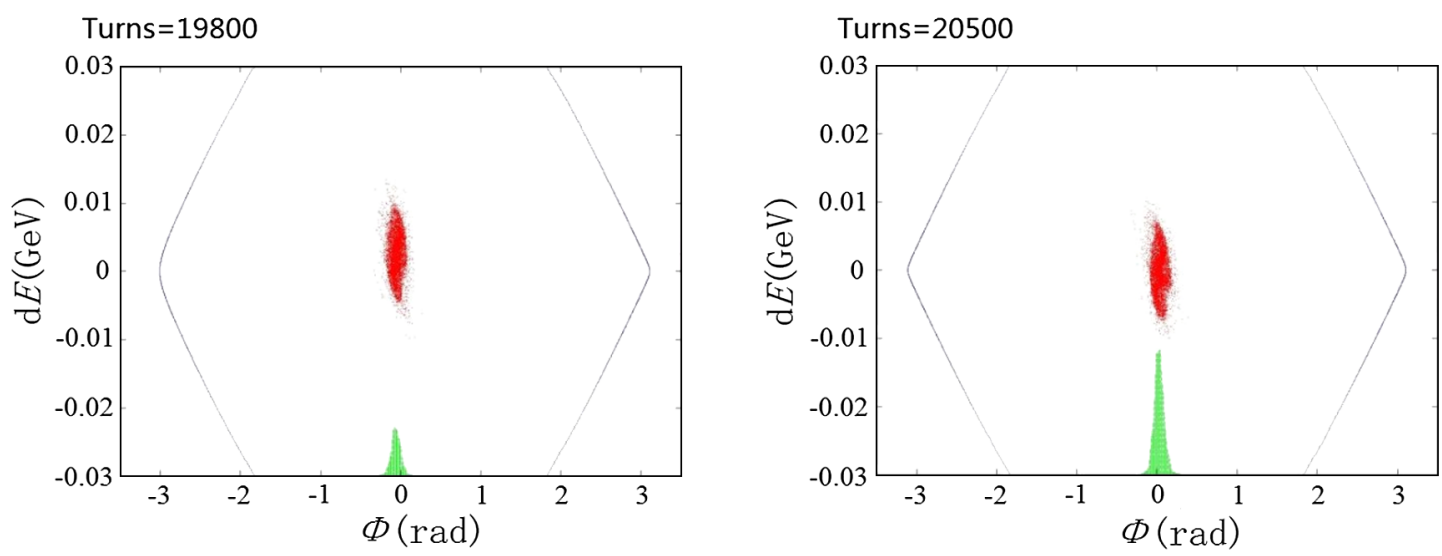

FIG. 5. Longitudinal beam distributions just before the extraction in the nominal operation mode with bunch compression (Left: without desynchronization; right: with de-synchronization.)
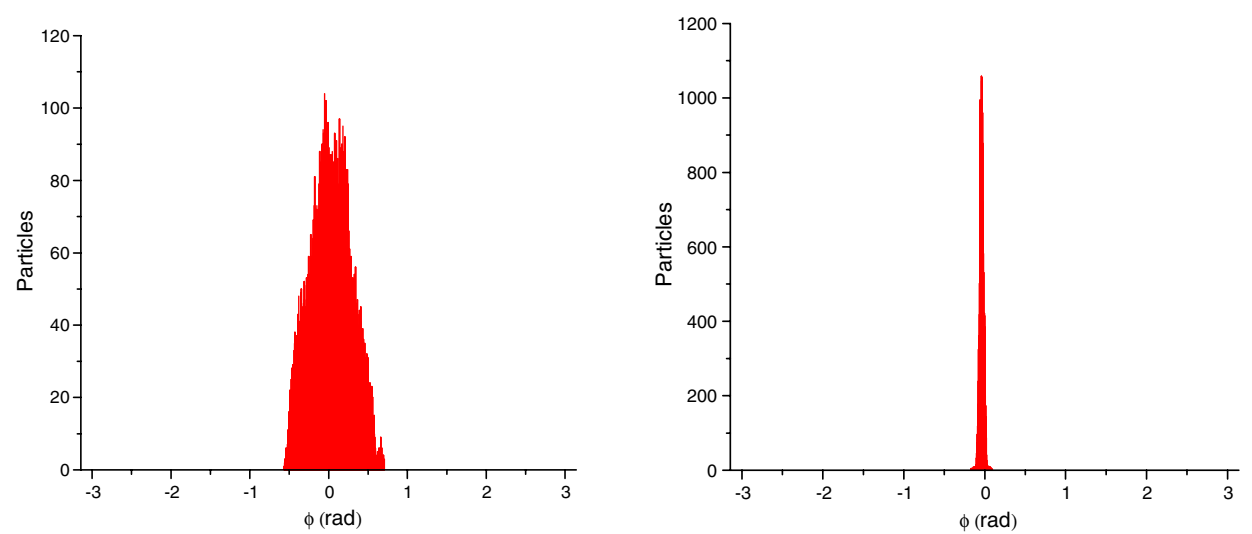

FIG. 6. Phase distributions of the extracted beam. (Left: in mode A; right: in mode D.)

During the last few turns of the bunch rotation which lasts about $0.2 \mathrm{~ms}$ or 200 turns when the beam becomes very short, the Laslett tune shift again becomes a concern despite the relatively high energy. Fortunately, the

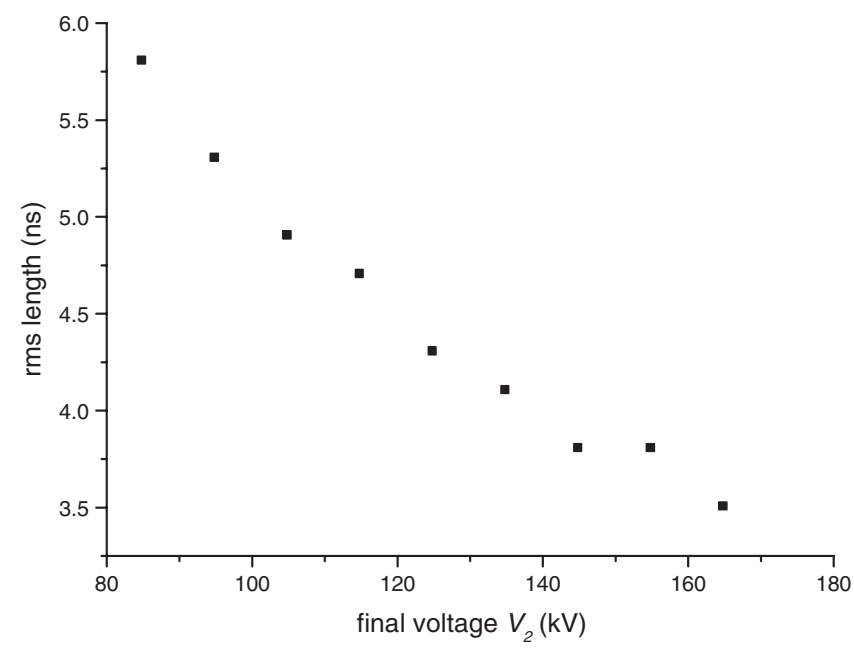

FIG. 7. The rms extraction bunch lengths with different final voltages in mode $\mathrm{B}$. calculations show that it is still within control, e.g. about -0.066 for mode $\mathrm{D}$, which is considered quite safe in such a high-intensity RCS.

Figure 3 shows the longitudinal beam distributions just before the extraction in the four modes described above. One can see that a much shorter bunch length can be obtained in the dedicated operation modes, especially with the injection mode D. There is a longitudinal beam halo formed during the injection and acceleration but it is not important for the users in neutron scattering and nuclear data measurement. For the neutron scattering application, the proton bunch length in the nanosecond level is not concerned; for the nuclear data measurements, what is important is to have a shorter rms bunch length. Figure 4 shows the bunch compression process in the case of mode D. Because of the short bunch lengthening time, there will be a slight bunch incline at the end of the adiabatic process. Figure 5 shows the longitudinal beam distributions just before the extraction in the nominal operation mode with bunch compression at the same time with or without applying the desynchronization method. One can see that the desynchronization is helpful for reducing the bunch length, especially when the longitudinal 


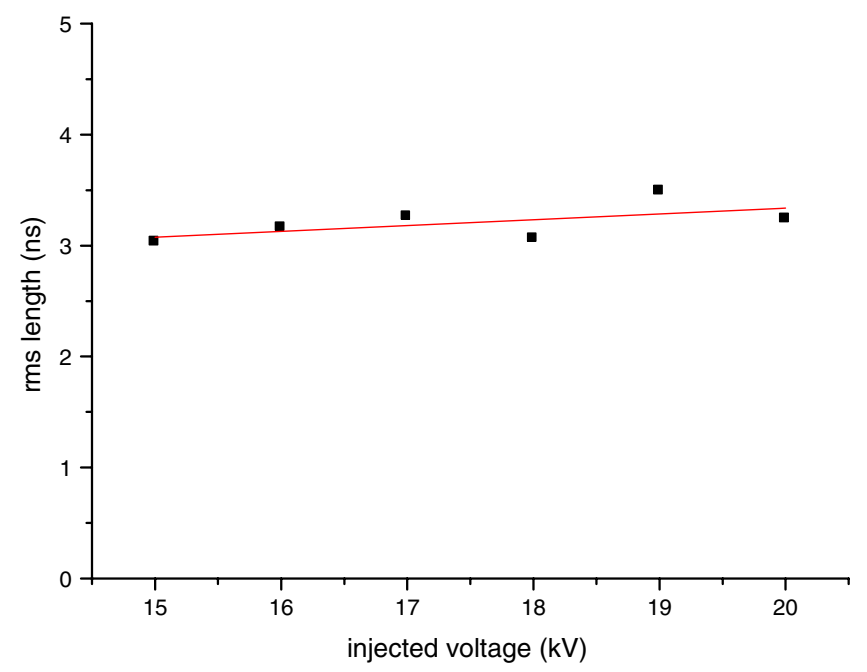

FIG. 8. The rms extraction bunch length with different injection voltages in mode $\mathrm{B}$.

emittance is large as in mode A where the rms bunch length was reduced from 7.5 to 3.9 ns. Figure 6 shows the phase distributions of the extracted beams in modes A and D. Figures 7 and 8 show the rms bunch lengths with different final voltages $\left(V_{2}\right)$ and different injected voltages. It can be seen that the higher final rf voltage helps reduce the rms bunch length of the extracted beam as expected. On the other hand, one can see that the rms bunch length is not sensitive to the injection voltage in the given range due to the emittance dilution as explained in the beginning of the section. For comparison, the rms bunch lengths for the extracted beams with different scenarios on injection and beam power reductions are shown in Table III. Combining the benefits from the bunch compression, smaller longitudinal emittance and desynchronization, mode $\mathrm{D}$ gives a much shorter bunch length or about one-ninth for the extracted beam than the one in mode A-the nominal operation mode. The bunch distribution of the extracted beam is very close to be Gaussian, as shown in Fig. 9. As mentioned before, the time structure of the neutron pulse has to include the contribution of the thick target at CSNS [21]. Figure 10 shows the time resolution of the

TABLE III. The rms bunch length with different chopping factors and beam powers.

\begin{tabular}{|c|c|c|c|c|c|}
\hline $\begin{array}{l}\text { Injection } \\
\text { mode }\end{array}$ & $\begin{array}{l}\text { Chopping } \\
\text { factor }(\%)\end{array}$ & $\begin{array}{c}\text { Beam } \\
\text { power } \\
(\mathrm{kW})\end{array}$ & $\begin{array}{c}\text { rms } \\
\text { length } \\
\text { without } \\
\text { BC (ns) }\end{array}$ & $\begin{array}{l}\text { rms length } \\
\text { with BC } \\
\text { without } \\
\text { DS (ns) }\end{array}$ & $\begin{array}{l}\text { rms length } \\
\text { with BC } \\
\text { with } \\
\text { DS (ns) }\end{array}$ \\
\hline A & 50 & 100 & 13.0 & 7.5 & 3.9 \\
\hline B & 50 & 50 & 11.0 & 3.5 & 3.3 \\
\hline $\mathrm{C}$ & 50 & 30 & 9.3 & 3.3 & 3.1 \\
\hline D & 30 & 30 & 7.2 & 1.7 & 1.5 \\
\hline
\end{tabular}

Note: BC denotes "bunch compression" and DS denotes "desynchronization."

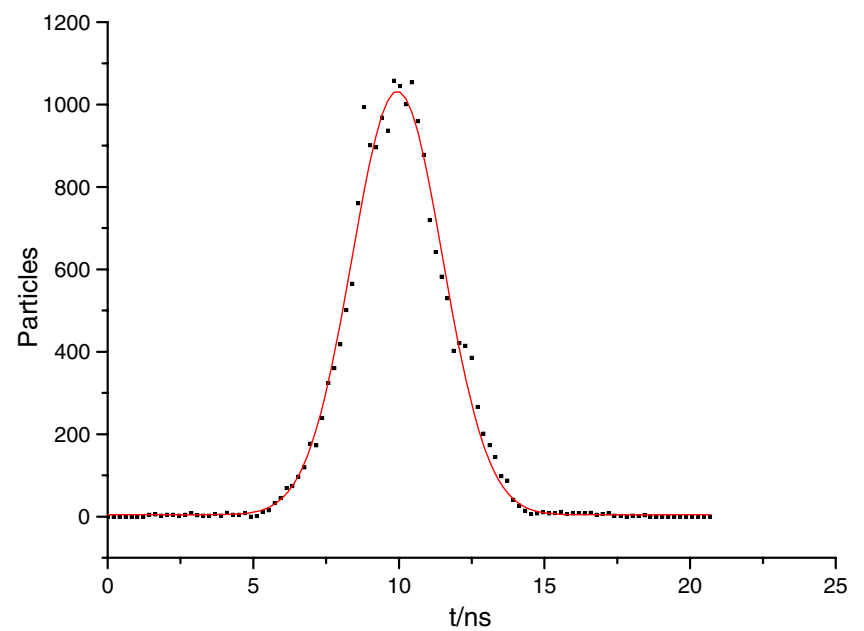

FIG. 9. Gaussian fit for a single extracted beam bunch in mode D.

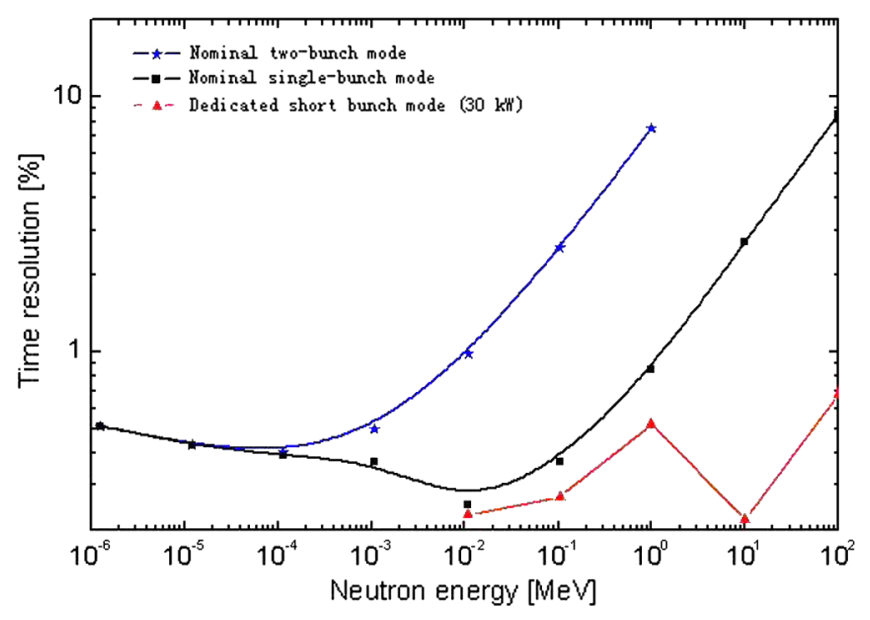

FIG. 10. Time resolutions of the backstreaming neutrons for the nuclear data measurements using the time-of-flight method.

backstreaming neutrons at a distance of about $80 \mathrm{~m}$ from the target for the nuclear data measurements using the timeof-flight method. One can see that using the short-bunch extraction method, we can improve the time resolutions significantly for neutrons with energy higher than $10 \mathrm{keV}$, and dramatically for neutrons with energy higher than a few MeV.

\section{CONCLUSIONS AND DISCUSSION}

The studies show that the bunch compression method is still effective in extracting short-bunch beams in rapid cycling synchrotrons, with the example of CSNS/RCS. A special desynchronization method is used here to avoid the slow acceleration during the bunch rotation process. Different injection/extraction scenarios or operation modes at the CSNS/RCS have been studied. In the dedicated case with a sacrifice of beam power reduction of $70 \%$, the rms bunch length is only one-ninth of the one in the nominal 
operation mode. With the reduced bunch length, the time resolution for the white neutron applications is improved dramatically for neutrons with energy higher than a few $\mathrm{MeV}$. Although the detailed studies have been carried out with the CSNS parameters, the method should be applicable to other high-intensity proton synchrotrons with high available rf voltage.

The study will continue in the future. The impedance effect will be included in the multiparticle simulations. As CSNS will be upgraded to $500 \mathrm{~kW}$ in a later phase when a dual harmonic rf system will be used, how to proceed the short-bunch extraction in this condition is needed to be studied. Although the reduction beam power in the dedicated WNS operation modes (modes B, C, D) is not very important at CSNS for a very limited operation time per year, it might be important for other high-power RCSs to obtain as high as possible a beam power along with very short bunches. Further studies are needed to push a higher beam power with a small chopping factor but higher linac peak current or more injection turns for different bunch lengths at extraction as the beam loading effect is taken into account in a quite conservative way in the present study.

\section{ACKNOWLEDGMENTS}

The authors would like to thank the CSNS colleagues for discussions. The study is jointly supported by the CSNS project and the National Natural Science Foundation of China (Projects No. 11235012 and No. 10975150).

[1] J. Y. Tang, in Review of Accelerator Science and Technology, edited by A. W. Chao and W. Chou (World Scientific Publishing, Singapore, 2013), Vol. 6.

[2] K. Blasche, O. Boine-Frankenheim, H. Eickhoff, et al., in Proceedings of the 6th European Particle Accelerator Conference, Stockholm, 1998 (IOP, London, 1998), pp. 1347-1349 [http://accelconf.web.cern.ch/AccelConf/ e98/PAPERS/THP41G.PDF].

[3] C. R. Prior, Proceedings of the European Particle Accelerator Conference, Vienna, 2000 (EPS, Geneva, 2000), p. 963 [http://accelconf.web.cern.ch/AccelConf/ e00/PAPERS/THP4A06.pdf].

[4] K. Y. Ng, Report No. FERMILAB-FN-0702, 2001.
[5] J. W. G. Thomason, R. Garoby, S. Gilardoni, L. J. Jenner, and J. Pasternak, Phys. Rev. ST Accel. Beams 16, 054801 (2013).

[6] Jie Wei et al., Nucl. Instrum. Methods Phys. Res., Sect. A 600, 10 (2009).

[7] H. T. Jing, J. Y. Tang, and Z. Yang, "Study on collimation and shielding of the back-streaming neutrons at the CSNS target" (to be published).

[8] J.-Y. Tang, S.-N. Fu, H.-T. Jing, H.-Q. Tang, J. Wei, and H.-H. Xia, Chin. Phys. C 34, 121 (2010).

[9] K. M. Fung et al., Report No. FERMILAB-Pub-00/198-E, 2000.

[10] S. Y. Lee, Accelerator Physics (World Scientific Publishing, Singapore, 1999).

[11] R. Cappi, J. Gareyte, E. Métral, and D. Möhl, Nucl. Instrum. Methods Phys. Res., Sect. A 472, 475 (2001).

[12] J. Pasternak, M. Aslaninejad, K. Long et al., Proceedings of the 23rd Particle Accelerator Conference, Vancouver, Canada, 2009 (IEEE, Piscataway, NJ, 2009), p. 1433 [http://accelconf.web.cern.ch/AccelConf/PAC2009/papers/ tu6pfp064.pdf].

[13] P. Spiller, J. Ahrens, K. Blasche et al., Proceedings of the 19th Particle Accelerator Conference, Chicago, IL, 2001 (IEEE, Piscataway, NJ, 2001), p. 3278 [http://accelconf .web.cern.ch/AccelConf/p01/PAPERS/RPPH024.PDF].

[14] D. Boussard, Report No. CERN 87-03, 1987.

[15] P. Barratt, I. Gardner, C. Planner, and G. Rees, Proceedings of the European Particle Accelerator Conference, Nice, France, 1990, p. 949 [http://accelconf.web.cern.ch/ accelconf/e90/PDF/EPAC1990_0949.PDF].

[16] Accelerator Technical Design Report for J-PARC, J-PARC 03-01, 2003.

[17] H.C. Liu et al., Proceedings of the 4th International Particle Accelerator Conference, IPAC-2013, Shanghai, China, 2013 (JACoW, Shanghai, China, 2013), p. 3351 [http://accelconf.web.cern.ch/accelconf/IPAC2013/papers/ thpfi025.pdf].

[18] J. F. Chen and J. Y. Tang, Chin. Phys. C 34, 1643 (2010).

[19] J. D. Galambos et al., ORBIT User Manual, Version 1.10, SNS/ORNL/AP Technical Note, 1999 [http://mad.home.cern .ch/frs/Source/space_charge/Literature/2011_Galambos.pdf].

[20] J. F. Chen, X. Y. Zhang, and J. Y. Tang, in Proceedings of HB2012, Beijing, 2012, pp. 487-491 [http://accelconf.web .cern.ch/AccelConf/HB2012/papers/tho1a03.pdf].

[21] H. T. Jing, J. Y. Tang, H. Q. Tang, H. H. Xia, T. J. Liang, Z. Y. Zhou, Q. P. Zhong, and X. C. Ruan, Nucl. Instrum. Methods Phys. Res., Sect. A 621, 91 (2010). 